\title{
Electronic windows
}

\author{
DAVID PETTIJOHN \\ Massachusetts Institute of Technology, Cambridge, Massachusetts 02139
}

\begin{abstract}
A circuit is described which allows the definition of target zones or windows for use in motor coordination experiments. The outputs of the circuit are logic levels and pulses which can be used to automate the experiments.
\end{abstract}

As part of our studies on motor coordination, we are using a paradigm that requires a monkey to position its arm or turn its head so that it is located within a target zone. These target zones, or windows, are defined electronically.

A typical experimental situation is shown in Figure 1 . A shaft, attached to the monkey's head via screws. allows the monkey's head to rotate in the horizontal planc only. The rotation of the head is measured by means of a potentiometer coupled to the shaft. Small lamps are set in front of the monkey along an arc of $1 \mathrm{~m}$ radius centered at the monkey's head. The monkey is trained to turn its head so that it is aligned with whichever lamp is on. The monkey is rewarded with water if it positions its head in a target conc and holds it there for $1 \mathrm{sec}$ within the 2 -sec trial.

A schematic of the geometry of the experiment is shom in Figure 2; the window circuit diagram is shown in Figure 3 . The position of the lamp defines the conter of the window: the width of the window is controlled independently.

Operation of the circuit is as follows: With the head lixture turned to face a lamp, the NULL potentiometer is set so that the output, $V_{a}$, of the summing amplifier, $A_{1}$, is equal to $0 \mathrm{~V}$. In general,

$$
\mathrm{V}_{\mathrm{a}}=-\left(\mathrm{V}_{\mathrm{NULL}}+\mathrm{V}_{\text {HEAD }}\right)
$$

$\mathrm{V}_{\text {HEAD }}$ is the voltage from the head potentiometer. ${ }^{1}$ If the animal's head is on one side of the lamp, $V_{a}$ will be negative; on the other side, $V_{a}$ will be positive. The width of the angle about the lamp, i.e., the width of the window, is set by the WIDTH potentiometer and the inverting amplifier, $A_{2}$. The voltage from this potentiometer and its negative $\left( \pm V_{B}\right)$ are applied to an input of $301 \mathrm{~A}$ amplifiers used as comparators. These voltages provide the boundaries of a voltage " indow with $0 \mathrm{~V}$ as the center. When $V_{\mathrm{a}}= \pm \mathrm{V}_{\mathrm{B}}$, the appropriate comparator will change state, and the transition is detected.

When the monkey turns his head toward the light, as the head enters the window, the head potentio-

Research supported by NIH under Grant NS09343. I would like to thank Mr. Andres Polit. M.1.T. Psychology Department, for his helpful suggestions and for the basic drawing of Figure 1.

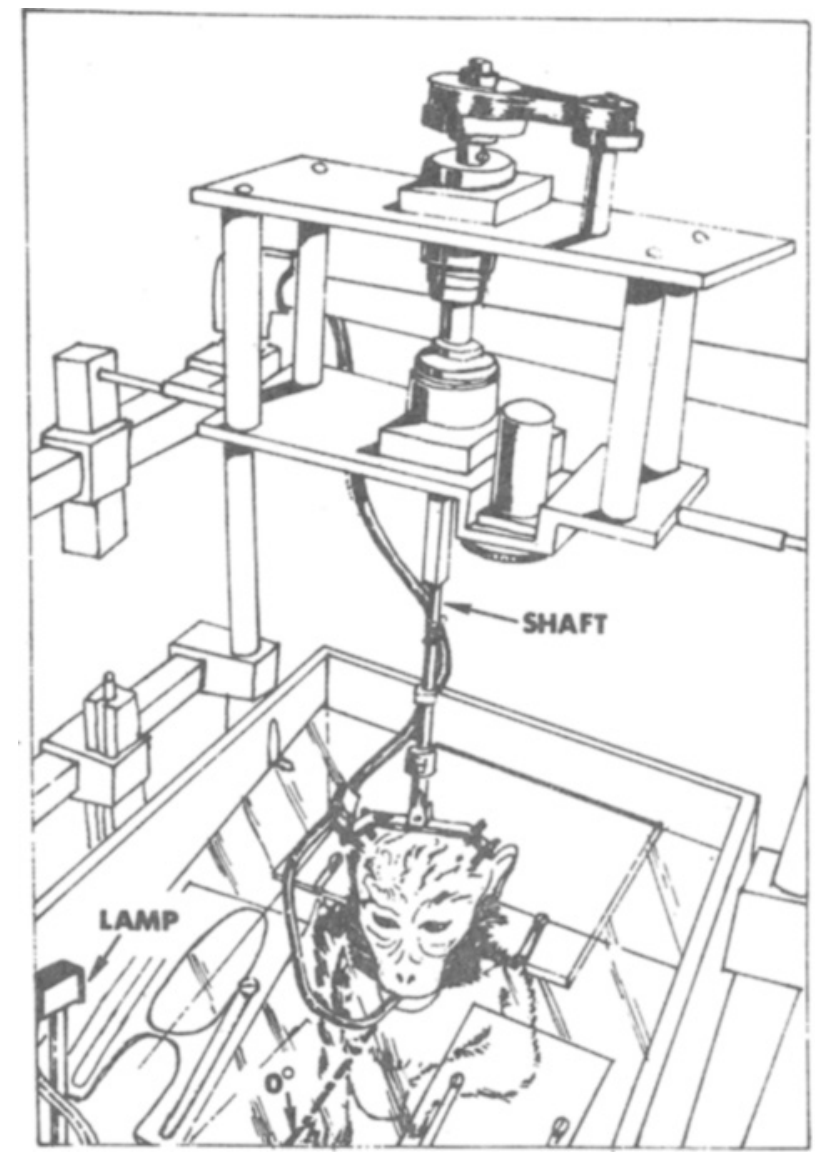

FIG. I

meter voltage crosses one of the boundary voltages, $\mathrm{V}_{\mathrm{B}}$, and a voltage transition occurs at the output of a comparator. A 5.1-V Zener diode connected to compensation Pin 8 of the $301 \mathrm{~A}$ constrains the comparator output to $0 \longleftrightarrow 4 \mathrm{~V}$ (National Semiconductor Corp., 1973), which is compatible with most digital circuits. The 7486 Exclusive-OR network produces a negative-going voltage spike (Electronic Design, 1974) for each comparator transition. Thus, a spike is generated whenever the animal's head crosses a window boundary. This spike is used to trigger 


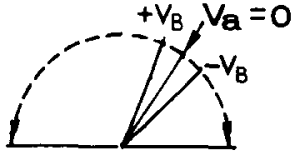

FIG. 2

recognition circuits, such as flip-flops (memory) and one-shots (timing intervals).

In the circuit of Figure 3, the spike outputs of both boundary comparators are ANDed together to trigger a 4123 one-shot whenever a boundary is crossed. When it is triggered. $\mathrm{R}$ and $\mathrm{C}$ are chosen such that its output changes from LO to HI for $140 \mathrm{nsec}$. (This interval is required for a PDP-11/40 computer input recognition.) The one-shot output, called BDRY $\mathrm{XING}$, is input to a PDP- $11 / 40$ computer as an interrupt signal.

Additionally, a unique logical state exists when the head is within the window; the output of both boundary comparators is $\mathrm{HI}$ in this case. In Figure 2,
Table 1

Integrated Circuits for Electronic Windows (Manufactured by Any Major Supplier)

\begin{aligned} & 310 $\begin{array}{l}\text { Voltage follower (National Semiconductor) } \\ \text { High-performance general-purpose operational ampli- } \\ 748 \text { (Motorola) }\end{array} \\ & 7486 \begin{array}{l}\text { Medium-speed TTL quad two-input Ixclusive-OR } \\ \text { gates (Texas Instruments) } \\ \text { Medium-speed TTL quad two-input AND gates (Texas }\end{array} \\ & 74123 \begin{array}{l}\text { Instruments) } \\ \text { Medium-speed TTL dual monostable multivibrator } \\ \text { (Texas Instruments) }\end{array} \\ &$\hline\end{aligned}

these outputs are ANDed to provide an "IN WINDOW" bit, which is also input to the computer as data. The circuit provides the precise points at which the animal's head entered or left a window in the experiment, as well as the time that the animal stayed in the window.

We use three electronic windows in our computer-to-laboratory interface system. ${ }^{2}$ In other

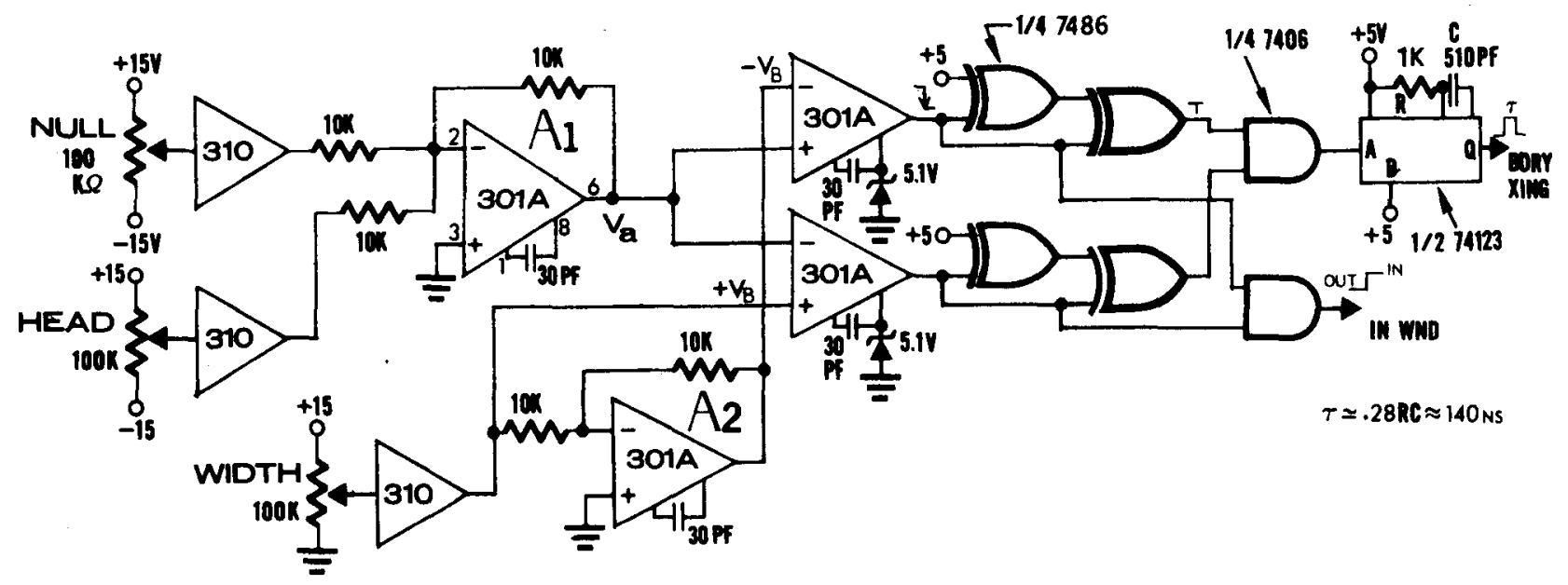

FIG. 3

angular or rotating contexts, such as arm pointing, mentioned earlier, a monkey's arm is held in a fixture which is free to rotate in a plane; when the lamp is lighted, the monkey is trained to point at the light by turning the arm fixture to the light.

With respect to components, 310 voltage followers are used to present low-impedance sources to the summing and width amplifiers. 301 $\mathrm{A}$ amplifiers are used because they are an inexpensive high-quality circuit with the added benefit of allowing access to the compensation terminals (pins 1 and 8 ). This feature is exploited in the logic-output comparators, and can be used to effect minor-loop feedback compensation for tailoring the frequency response of the amplifier. With $30 \mathrm{pF}$ capacitor compensation, performance is similar to a 741-type operational amplifier. Table 1 gives a parts list for the window circuit. Approximate cost is less than $\$ 40$. In fact. 310 voltage followers are relatively more expensive due to their extended bandwidth capability. 741 or $301 \mathrm{~A}$ operational amplifiers configured as unity-gain followers would be acceptable.

\section{REFERENCES}

National Semiconductor. Line'd integrated circuits data bork. Santa Clara. Calif: National Semiconductor Corporation. January 1973.

Fifrtronic Design, Voltage spikes generated from logic transitiums. Elactromic Design, 1974, 13. 11 h

\section{NOTES}

1. The potentiometer resistances should generally be greater than $10 \mathrm{k} \Omega$ for small power consumption. The exact choice depends on the arc of travel and the sensitivity required. We use linear. 10-turn. $5 \%$ tolerance potentiometers for NULL and WIDTH.

2. A paper describing the coniputer-laboratory interface system is in progress.

(Recerved for publication February 17. 1975: revision receined Marsh 10. 1455. 